\title{
Adoption of Cooperative Intelligent Transport Systems in Flemish passenger cars: a review of European Policy Options
}

\author{
Thibault Degrande \\ IDLab \\ Ghent University - imec \\ Ghent, Belgium \\ thibault.degrande@ugent.be
}

\author{
Frederic Vannieuwenborg \\ IDLab \\ Ghent University - imec \\ Ghent, Belgium \\ frederic.vannieuwenborg@ugent.be
}

\author{
Sofie Verbrugge \\ IDLab \\ Ghent University - imec \\ Ghent, Belgium \\ sofie.verbrugge@ugent.be
}

\author{
Didier Colle \\ IDLab \\ Ghent University - imec \\ Ghent, Belgium \\ didier.colle@ugent.be
}

\begin{abstract}
Cooperative Intelligent Transportation Systems (CITS) enable vehicles to be aware of objects that are not in line of sight, by interacting directly with each other and the surrounding road infrastructure. C-ITS promise to reduce traffic congestion, lessen the environmental impact of transportation, and most importantly, significantly reduce the number of (lethal) traffic accidents. As C-ITS is subject to strong network effects, adoption of C-ITS is a key driver of its (societal) benefits. Therefore, this paper estimates penetration rates of C-ITS equipped cars in the car park of Flanders, Belgium. Based on the preferred policy option with mandatory adoption, as proposed in the recent new Delegated Regulation of the European Commission, full penetration of C-ITS in the Flemish car park is expected later than twenty years after the mandate enters into force. Determination of C-ITS adoption numbers is valuable for a number of stakeholders, such as national and local governments, road authorities, technology providers and network operators. Additionally, penetration numbers allow Member States to ascertain to what extent C-ITS can contribute to the goal of zero traffic mortalities by 2050, as envisioned by the European Commission.
\end{abstract}

Index Terms-Cooperative intelligent transport systems, road safety, adoption, smart mobility

\section{INTRODUCTION}

$\mathbf{T}$ HE automotive industry is facing a technological revolution; apart from electrification and shared mobility concepts, technological advances will allow for autonomous driving in the decade to come. Autonomous Driving (AD) can, apart from powertrain electrification and car sharing initiatives, contribute to reduce the usage of fossil fuels and subsequently its negative environmental impact, mainly by increased traffic efficiency [1]. More importantly, AD has the potential to dramatically improve road safety by reducing driver errors. This is a great opportunity for the European Union (EU) and its Member States to realise the EU's long-term goal of moving close to zero fatalities and serious injuries by 2050 ("Vision Zero") [2]. As part of that vision, starting from 2010, the EU

This work is partially supported by the European CONCORDA project (Connected Corridor for Driving Automation, CEF Action 2016-EU-TM0327-S), as well as by the Belgian / Flemish SErVo project (Secure and Economically Viable V2x solutions, AH.2018.092). road safety guidelines aimed to cut European road deaths by $50 \%$ by 2020 [2]. Although different initiatives at the local, national and EU level have led to considerable progress since 2011 1 , reaching the objective of halving road fatalities will be very challenging [3].

The Flemish government, in particular, also acknowledges that it wil not achieve its intermediate 2020 objectives [4]. In a concept nota [4], the minister of mobility states that Flanders supports the aforementioned 'Vision Zero' and explicitly emphasises that the current developments in Connected, Cooperative and Automated Mobility (CCAM) will contribute and be used to achieve these objectives. Therefore, the Flemish government has engaged itself in this CCAM mobility (r)evolution by making it one of its transition priorities [5]. To this end, the Flemish government has participated in multiple research initiatives in this domain 2 Furthermore, in order to facilitate transition in the automotive sector and the use of CCAM equipped cars, the Flemish government underlines the importance of a European or global approach to tackle the many challenges related to C-ITS deployments [4].

While Intelligent Transport Systems (ITS) focused on providing intelligence at the roadside or in vehicles by digital technologies, Cooperative ITS focuses on the communication between those systems. It enables vehicles to interact directly with each other and the surrounding road infrastructure, allowing road users and traffic managers to share information and use it to coordinate their actions [6]. This communication involves vehicle-to-vehicle (V2V), vehicleto-infrastructure (V2I) communication, and communication between vehicles and pedestrians or cyclists (vehicle-toeverything (V2X)). By adding the communication aspect, CITS extends the vehicle's abilities by providing 360-degree non line-of-sight (NLOS) awareness. This enables a wide range of information and cooperation services [7]. Based on the work

\footnotetext{
149 road deaths per million inhabitants in 2017, compared to 63 in 2010, a decrease of $22 \%$

${ }^{2}$ The Mobility and Public Works department of the Flemish government is involved in a number of complementary European C-ITS projects, including CITRUS, InterCor and C-Roads.
} 
of the C-ITS Platform [8], the Commission has agreed on a list of technologically-mature and highly beneficial C-ITS services that should be deployed first, defined as the Day 1 C-ITS services [9]. These involve two broad categories, being (1) hazardous location notification (e.g. road works warning) and (2) signage applications (e.g. in-vehicle speed limits). In a second phase the Day 1.5 C-ITS services would be deployed, a list of services for which full specifications or standards might not be completely ready for large scale deployment. Important to notice is that these services encompass both road safety and traffic efficiency services, as the latter is another major concern in areas with high traffic density, such as Flanders.

The transition towards connected mobility is inherently intertwined with the development path towards automated driving. It is clear that cooperation, connectivity, and automation are not only complementary technologies; they reinforce each other and will over time merge completely [9]. The technologies are complementary, since communication between vehicles, infrastructure and other road users is crucial to increase the safety of future automated vehicles and their full integration in the overall transport system. Makridis et al. [10] even have quantitatively showed that automation without connectivity could lead to a potential deterioration of traffic conditions. In other words, connectivity and cooperation are prerequisites to safe automation [6]. Hence, as C-ITS is an essential intermediate step towards CCAM, its success greatly determines the introduction and successfullness of future CCAM.

To ensure that C-ITS success, the European Commission has published Delegated Regulation supplementing Directive 2010/40/EU of the European Parliament and of the Council, in order to create legal requirements for C-ITS interoperability between large scale deployments as from 2019. In this Delegated Regulation, the European Commission stipulates that maximising benefits involves leveraging the 'hybrid communication' approach. For short-range (i.e. V2V, V2I) communication, this hybrid combination makes use of ITSG5, a European standard for vehicular communication based on the p-amendment of IEEE 802.11 [11], while for long range communication (i.e. Vehicle-to-Network (V2N)), cellular connectivity is chosen. The choice for ITS-G5 is remarkable, as a technology battle had originated since June 2017, as the 3rd Generation Partnership Project (3GPP) at that time officially published long term evolution (LTE) release 14, in which it introduced the support of V2X services in the LTE standard [12]. This cellular alternative is referred to as Cellular-V2X (C-V2X), and has a clear evolution path towards 5G NR-based C-V2X. However, the Commission states that a review clause will facilitate the integration of existing candidates, such as $\mathrm{C}-\mathrm{V} 2 \mathrm{X}$ and $5 \mathrm{G}$ [7]. Besides the fact that the two competing short range technologies are at different levels of maturity and commercialisation, they are not interoperable at radio access level. The latter is an important issue for several reasons. First, interoperability is a key element in the European C-ROADS vision: it is unacceptable that lethal traffic accidents would be caused by non-interoperable communication systems [13]. Furthermore, as they reside at the same $5.9 \mathrm{GHz}$ frequency, they could cause harmful interference to one another. Next, it is not costeffective to equip roadsides with two competing technologies that serve the same purpose. Finally, C-Roads Member States are committed to the "backwards compatibility" criteria in the technological evolution [13]. As several Member States already started the procurement and deployment of $802.11 \mathrm{p}$ based equipment, new C-ITS equipment beyond Day-1 needs to support and safeguard already deployed C-ITS services.

The driver of the envisioned benefits of C-ITS and subsequently, CCAM, is the extent to which cars and roads are equipped with C-ITS systems. This is because in C-ITS, the road user perceives only a benefit, if the other vehicle(s) in near vicinity (300-400m distance) are also equipped with a C-ITS station. Since C-ITS is thus subject to strong network effects, the penetration rate of vehicles equipped with direct communication is highly critical for the effectiveness of $\mathrm{V} 2 \mathrm{~V}$ use cases. Therefore, this paper looks at penetration rates for passenger cars with C-ITS On-Board-Units (OBUs) in Flanders, in the light of European policy options depicted in the Delegated Regulation. Section $[\mathrm{I}$ presents an overview of related work on technology adoption. Next, Section III states the different assumptions made to estimate the adoption curves depicted in Section IV] Finally, Section V concludes.

\section{RELATED WORK ON ADOPTION}

Policy incentive schemes can advance technology adoption, as consumers may be reluctant to pay for the price premium of vehicles equipped with C-ITS stations. The reluctancy for paying the premium has many potential reasons, including the well-known observation that consumers overvalue the price at purchase time with respect to benefits or savings on the long term. For instance, C-ITS promises to improve traffic efficiency and therefore will reduce fuel consumption [8]. However, Green et al. [14] indicate that the rational economic model, in general, does not appear to be used by consumers when comparing the fuel economy of new (in this case, CITS equipped) vehicles. Next, [15] states that consumers also do not value the safety benefits (in particular not for the society as a whole) of additional safety equipment. However, other aspects such as brand trust and privacy concerns can also be barriers to adoption. Probably, it will take prove that the technology can be used safely and reliably in real-world conditions, to make consumers feel more comfortable about these new technologies [16]. As a consequence, deliberate choice of C-ITS features by consumers will probably not lead to the envisioned rapid C-ITS adoption.

\section{A. Mandated adoption}

Stakeholders such as local governments or Original Equipment Manufacturers (OEMs) might only consider investments in the relevant infrastructure and hardware (e.g. Road Side Units (RSU)) once a substantial market penetration rate has been obtained. The European Commission has, in this regard, 
provided a substantial amount of compensation for infrastructural investments to its members states and partners, to overcome this barrier 3

Apart from policy incentive schemes, mandatory regulations can also be used to enforce adoption. For instance, with regard to eco-innovation adoption, complying with regulations is one of the most important motivations [17]. Furthermore, Zhou et al. [18] discuss studies that confirm the positive role of regulations in smart meter deployments, as countries with mandatory regulations tend to be leaders. More related to traffic, the European Parliament adopted new measures to improve road safety and reduce accidents in April 2019, making a number of safety features compulsory in new cars [19].

With regard to C-ITS, the choice of the short-range wireless technology to enable vehicular connectivity is a crucial issue. Since the unfolding will mostly depend on mandatory laws at the international level, the Commission repeatedly expressed the need for regulation to ensure data standardisation of vehicle communication protocols across different brands. In the EU strategy toward automated mobility [20], the Commission further stressed the importance of the ability of vehicles to communicate in integrating automated vehicles in the overall transport system. Therefore, the European Commission has released a Delegated Regulation on March 13 2019, supplementing Directive 2010/40/EU of the European Parliament and of the Council with regard to the deployment and operational use of cooperative intelligent transport systems [7], in which it motivates a stepwise approach towards a vehicle-to-vehicle (V2V) mandate. This stepwise approach includes the adoption of legally binding specifications ensuring all new vehicles being equipped with C-ITS stations, and additional measures that support the deployment of C-ITS. Analogous to the new rules on safety features, the European Commission first proposed three policy options [21]. The V2V mandate policy option was considered to be the most coherent and effective, delivering the highest reductions in accidents, congestion and $\mathrm{CO}_{2}$ emissions, of all three examined policy options (PO) [7]. The first policy option (PO1) represented light intervention based on non-legislative measures such as guidelines, memoranda of understanding (MOUs), stakeholder coordination or knowledge exchange platforms. This is in line with the current support via the C-ITS platform of the European Commission. Regarding communication specifications, PO1 would refer to existing standards on interoperability and EU-wide service profiles in guidelines. PO2 entailed moderate intervention, with elements similar to those in PO1, but legally binding through a Delegated Regulation. Compliance with this Delegated Regulation would be mandatory only when deploying C-ITS services or stations, but Member States and industry remain free to decide whether or not to deploy C-ITS [7].

\footnotetext{
${ }^{3}$ Connecting Europe Facility (CEF) for Transport supports innovation in the transport system in order to improve the use of infrastructure, reduce the environmental impact of transport, enhance energy efficiency and increase safety. The total budget for CEF Transport is 24.05 billion EUR for the the period 2014-2020 (https://ec.europa.eu/inea/en/connecting-europe-facility/ceftransport).
}

Finally, PO3 consists of a Vehicle to Vehicle (V2V) mandate for deployment of C-ITS that begins in 2021, on top of the the Delegated Act of PO2. It is assumed that a mandate in PO3 would not be introduced until 2021, given the common lead-in time for such a mandatory measure [22].

In the following section, the expected effect of the different policy options on the penetration of C-ITS equipped vehicles in the vehicle park of Flanders, Belgium will be discussed. First, the next subsection provides a brief overview of studies that have earlier estimated OBU C-ITS adoption in new cars, as C-ITS uptake in new cars will be a key input parameter in $\mathrm{C}$-ITS penetration in the Flemish vehicle park.

\section{B. Studies on C-ITS adoption in new cars}

In order to make the right policy option decisions, the European Commission has tried to assess the adoption of C-ITS equipment in vehicles, as input to the Cost-Benefit Analysis (CBA). In 'Study on the Deployment of C-ITS in Europe: Final Report' [8] from 2016, uptake and penetration rates for in-vehicle ITS sub-systems for new vehicles were defined separately for the three vehicle types modelled in the CBA, i.e. passenger cars, freight vehicles and buses (Annex E). Furthermore, they look to the uptake of C-ITS services in aftermarket systems, though aftermarket devices are focussed on offering $\mathrm{V} 2 \mathrm{~N}$ services only. They are assumed to not have V2V (ITS-G5) capability, due to the need for many of these services to be connected to the CAN bus of the vehicle [8]. Within the passenger cars, for some scenarios a distinction is made in segments, as the more upper segments are reached first. For Day 1, V2V services (ITS-G5), the deployment assumptions for in-vehicle ITS sub-systems for new vehicles lead to a baseline of reaching all Luxury cars (Upper Medium and Executive cars) by 2027, starting 2020. Different adoption scenarios lead to deployment in all Upper Medium and Executive cars by 2023 (2021), starting 2018, all Lower Medium cars by 2024 (2022), starting 2019 (2018), and all Small cars by 2025 (2023), starting 2020, for low (central/high) sensitivity, respectively.

In 2017, the 5G Automotive Association (5GAA) released its study on 'An assessment of LTE-V2X (PC5) and 802.11p direct communications technologies for improved road safety in the EU'. Annex B is dedicated to C-ITS technology penetration, in which the two technologies are considered independently and in isolation; i.e., where only LTE-V2X (PC5) or only ITS-G5 (802.11p) is deployed, up to the year 2040. Two scenarios are depicted, being 'low' and 'high' scenario. The 'low' scenario of $802.11 \mathrm{p}$ penetration results in $100 \%$ penetration in new cars by 2032, starting in 2019. In the 'high' scenario, a refresh cycle of 6 years for most vehicle models is assumed, yielding penetration rates in new cars of $100 \%$ in 2025 already. Penetration across the entire fleet is estimated by substracting cars that have exceeded the maximum lifetime of 14 years from the cumulative penetration function.

Finally, in order to assist the European Commission in developing a European framework to enhance the widespread 
deployment of C-ITS services (the Delegated Regulation supplementing Directive 2010/40/EU [7]), a 'Support study for Impact Assessment of Cooperative Intelligent Transport Systems' was was conducted [22]. This report from December 2018 projects uptakes in different scenarios, being (1) in the absence of further action beyond existing EU policy (baseline) and (2)-(4) under the different policy options described above from [7]. In this impact assessment, the uptake assumptions are mainly based on two concepts in car life cycles: (1) full model cycles and (2) facelift cycles. Full model cycles involve vehicle redesigns and are assumed to be 7 years for personal transport vehicles. Facelift cycles comprise minor upgrades to vehicle functionality and styling that occur midway through a model's lifecycle, and take 4 years [22]. In the first scenario, the baseline, uptake across new passenger cars reaches $100 \%$ in 5 vehicle full model cycles (35 years), starting in 2019. For PO1, the maximum penetration in new cars is reached after 4 full model cycles (28 years), starting 2019. Furthermore, according to the study, $\mathrm{PO} 2$ would lead to this penetration in 4 facelift cycles (16 years), and $\mathrm{PO} 3$ expects the mandate to reach all new cars in one full model cycle (7 years), starting in 2021. Analogous to the 5GAA study, vehicle scrappage is taken into account when looking at the total cumulative amount of new C-ITS equipped cars, with an average lifetime of vehicles across the EU of 12 years.

\section{APPROACH}

In this section, the focus is on adoption of C-ITS stations in passengers cars only, as the current Flemish testbed currently entails highway use cases for passenger cars. As public transport is not present on highways, also this transport category is not in scope of this study, nor does the category of Vulnerable Road Users (VRU, e.g. pedestrians). Furthermore, the costs related to C-ITS equipment in vehicles amounts to $90 \%$ of the total deployment costs [22]. In addition, most of Day-1 services can be implemented without RSUs, therefore, this paper does not consider the uptake of RSUs. Hence, the dependent variable of interest is OBU penetration rate in the passenger car park in Flanders, while the independent variables are the European policy options depicted in [7].

The penetration of C-ITS equipped cars in Flanders used in this paper for a given year $\mathrm{i}$ is presented in (1):

$$
P_{t, i}=\frac{\left(E_{t, i-1}-E_{r i}+E_{n i}\right)}{C_{t, i}}
$$

With:

1) $C_{t, i}$ : the total amount of cars for year i. Based on the data of Statistiek Vlaanderen 4 , the linear trend for the number of cars in Belgium from 1990 until 2016 is used to forecast the total amount of cars in Belgium. This number is validated by calculating the number of cars per 1,000 inhabitants, as Statistics Belgium (StatBel) has made predictions on the population of Flanders until the year 2071 $\sqrt{5}$. Furthermore, $C_{t, i}$ must

\footnotetext{
${ }^{4} \mathrm{http}: / /$ statistieken.vlaanderen.be/

${ }^{5} \mathrm{https} / / /$ statbel.fgov.be/nl/themas/bevolking/bevolkingsvooruitzichten\#panel-
}

be equal to $\left(E_{t, i-1}-E_{r i}+E_{n i}\right)+\left(N_{t i-1}-N_{r i}+N_{n, i}\right)$, the sum of the number of C-ITS equipped and non-equipped vehicles for a given year i.

2) $E_{t, i}, N_{t, i}$ : amount C-ITS equipped cars and non-C-ITS equipped cars in year $\mathrm{i}$, respectively.

3) $E_{r, i}, N_{r, i}$ : the scrappage vehicles for equipped and non-equipped vehicles in year i. FEBIAC 6 the Belgian and Luxembourg federation for automobiles, has data from 1985 until 2017 on the total amount of cars that are removed from the Belgian car park. In relative numbers, this amount is rather constant, at around $8.5 \%$ per year of the total fleet. An equal rate for the complete car park in Flanders is assumed. For the removal of C-ITS equipped cars after their introduction in the Flemish car park, a scrap function based on vehicle age is deducted from the data depicted in Fig. 11 It shows the distribution of the Belgian vehicle park according to the age of the vehicles in the years 2014 up to 2018 .

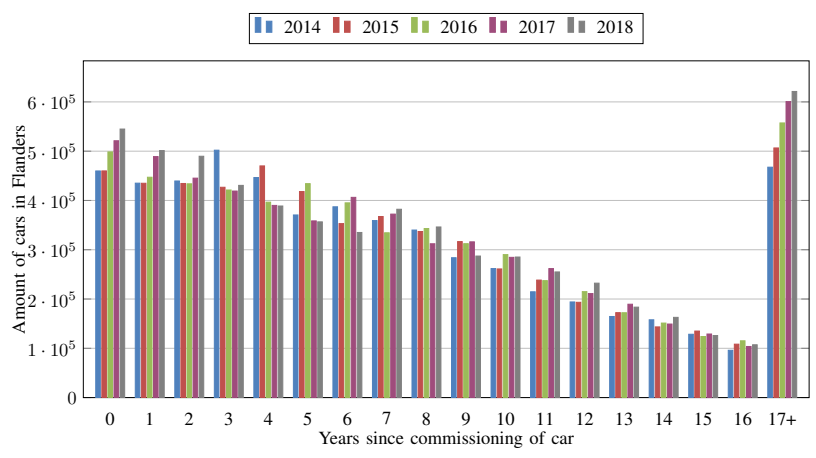

Fig. 1: Fleet composition by vehicle age (Febiac)

4) $E_{n, i}, N_{n, i}:$ amount of new car inscriptions for equipped and non-equipped vehicles in year $i$. The sum of these numbers is obtained by combining the predictions of the total car park size and the scrappage vehicles for a given year i. $E_{n, i}$ is calculated as an (increasing) percentage of total new cars inscripted. The growth rate of that percentage is dependent on the policy option. There are reasons to believe that adoption will start in the years to come, even with light intervention. Apart from existing regional and national deployment projects, several car manufactures have made announcements on equipping cars with C-ITS substations. For instance, Volkswagen already promised to enable vehicles to communicate with each other as from 2019 [23]. Renault is also developping C-ITS equipped cars, and has produced 1,000 enabled Mégane cars for the French Scoop project. Toyota and Lexus [24] have also announced to launch connected vehicles in 2021. Volkswagen, Renault, Toyota and Scania are strong proponents of the ITS-G5 communication standard. On the other side of the communication technology battle, the release of commercially available $\mathrm{C}-\mathrm{V} 2 \mathrm{X}$ chipsets and the numerous (successful) field tests of car manufacturers such as BMW, PSA groupe and Ford indicate the production of C-ITS equipped cars from these OEMs, too. Ford was the first to announce at CES 2019 a time-

\footnotetext{
${ }^{6}$ https://www.febiac.be/public/statistics.aspx ?FID=23\&lang=NL
} 
line for introduction of $\mathrm{C}-\mathrm{V} 2 \mathrm{X}$ communications beginning in calendar year 2022 [25]. In the case of non-binding guidelines, we can expect these OEM to contiunue on the C-V2X path. Data on the inscriptions of new cars in Belgium (FEBIAC) reveal that the ITS-G5 adepts (Volkswagen (9.2\%), Renault (9.3\%), Toyota (3.2\%) and Lexus $(0.3 \%)$ ) accounted for $21.6 \%$ of new cars in 2017, while C-V2X votaries (Audi (6.1\%), Ford $(4.5 \%)$, BMW $(9.1 \%)$, PSA groupe $(20.6 \%)$ ) accounted for $38.8 \%$. This means that over $60 \%$ of cars of new inscriptions stem from OEMs willing to move forward with C-ITS on the short term. However, not all models will be equipped from the earliest years, and the interoperability issue between the two standards will most likely make car manufacturers reluctant to go forward until the market finally completely settles for one standard. Furthermore, [26] reports that the transition between the 'innovator and early adopter' phase and the broader 'majority' take up phase of ITS deployment is often the hardest and most complex. Therefore, a light intervention is not the preferred option of the European commission. For all POs, uptake of C-ITS equipment in new cars is taken from [22], although shifted by one year for PO1 and PO2, as this work assumes the uptake to start in 2020.

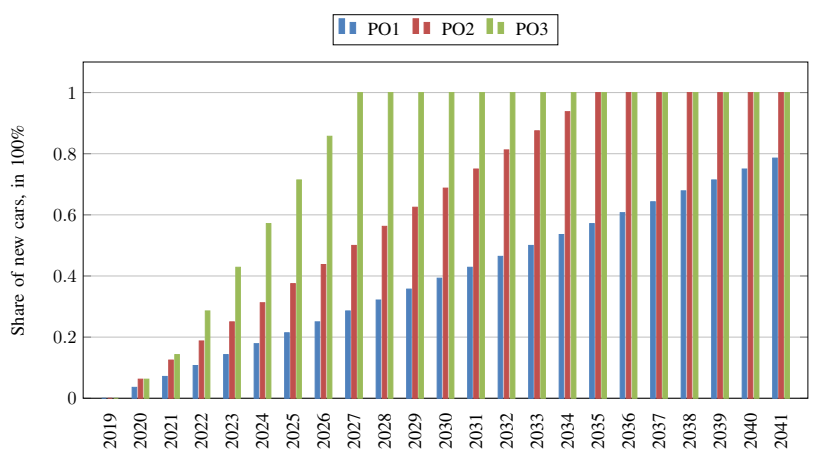

Fig. 2: Uptake of C-ITS stations in new cars, from [22

An overview of the uptake rates of C-ITS in new cars under the different policy options is given in Fig. 2. Year range from 2019 to 2041, twenty years after 2021, the expected year that a V2V mandate would go into force.

\section{RESUlTS AND Discussion}

Fig. 3 gives an overview of the total amount of cars in Flanders equipped with C-ITS. While Fig. 4 gives the penetration of C-ITS cars in the Flemish car park. Note that the uptake numbers for new vehicles are based on mandating for every new vehicle type-approval. Other introduction scenarios, such as mandating for every sold vehicle, would lead to faster C-ITS diffusion. For instance, the European Parliament mandated safety features such as intelligent speed assistance and advanced emergency-braking systems to be installed in new vehicles from May 2022 and in existing models from May 2024. In this work however, no aftermarket is considered, due to the need for many of the $\mathrm{V} 2 \mathrm{~V}$ services to be connected to the CAN bus of the vehicle.

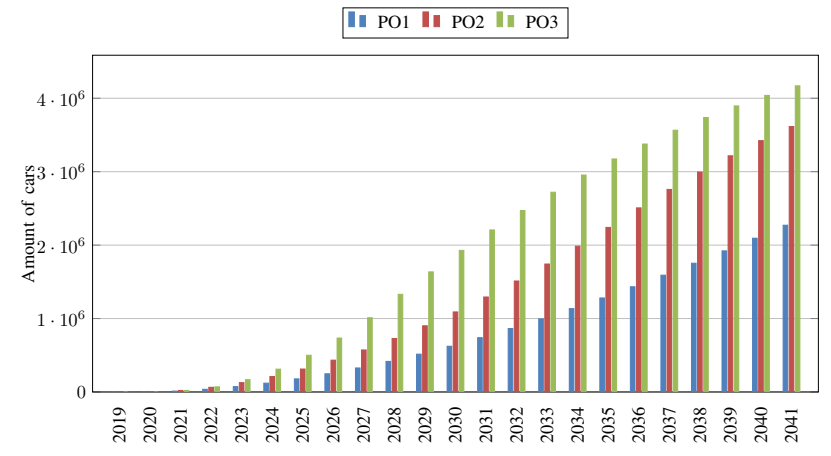

Fig. 3: Total cumulative amount of C-ITS cars in Flanders

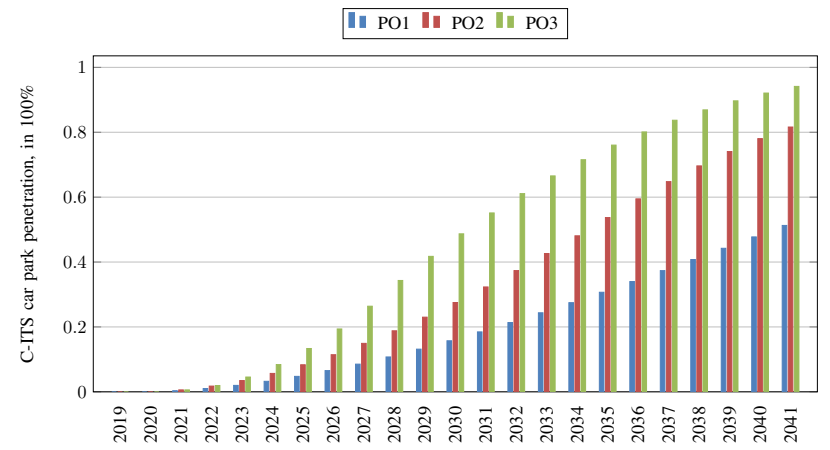

Fig. 4: Penetration of C-ITS cars in Flemish car park

The results are in line with [27], that stated that a penetration rate of $20 \%$ is realistic between 6-8 years from the point of starting the delivery of sidelink-equipped vehicles. $20 \%$ is the penetration rate for which the first effects of a direct communication technology are perceived [27]. Evidently, PO3 yields the best results in terms of penetration. One can see that under PO3, the total amount of C-ITS equipped cars is surpassing 4 million vehicles by 2041 , corresponding to a penetration of $95 \%$. For completeness, an equal rate of penetration via an every vehicle sold-mandate would be obtained in 2037 (i.e. $100 \%$ of new cars have to be C-ITS equipped as of 2021). However, this scenario is not equally realistic, because the thorough impact of implementing C-ITS stations in all models is likely to take a car model life cycle, and is therefore not further looked into.

The methodology has a few shortcomings. First, it assumes a continuation of the current predominantly private car ownership. This assumption is defensive, as it might be expected that car sharing initiatives would (1) lead to higher vehicle rotation and thus quicker C-ITS adoption in the shared car fleet, and (2) could lead to an accelerated scrap vehicle rate in non CITS cars, assuming that people get rid of their car in favour of a car-sharing subscription. Next, the model does not take into account possible factors that could negatively influence C-ITS adoption, such as malfunctions, abuses, malware or negative externalities. A first reason is that plenty of research is currently done in the domain, and that C-ITS equipment must obtain a declaration of conformity in order to assure meeting 
all requirements. To that end, the European Commission has established a C-ITS certificate policy authority, as stated in [7]. A second reason is that the European Commission will opt for the $\mathrm{V} 2 \mathrm{~V}$ mandate, so there is no room for OEMs to deliberately not adopt to C-ITS because of their reluctance by such possible negative effects.

Although numbers are only valid for Flanders, the methodology can be valuable for other stakeholders outside of Flanders, too. First, policy makers and local governments can determine if the efforts of Europe need additional measures. An analogy can be made with the Directive 2014/94 [28] on alternative transport fuels. There, Flanders biggest cities took additional initiatives on top of the (lacking) national policy framework, with the introduction of Low Emission Zones in Antwerp, Brussels and recently, Ghent. Thus, the Flemish government or local governments could set additional measures to enforce adoption, such as C-ITS zones, in analogy with Low Emission Zones. Second, adoption rates act as a driver of societal benefits, hence they serve as an input to costbenefit analyses for governments planning to deploy roadside units in urban or inter-urban setting. Next, governments, but also technology providers and network operators, can use adoption rate numbers to dimension the technical solutions. For instance, estimating data loads on the network, and the amount of data to process in Mobile Edge Computing Units or in central servers, for purposes such as traffic management. Finally, European Member States can use C-ITS adoption numbers to estimate the contribution of C-ITS in decreasing traffic mortalities.

\section{CONCLUSION}

As Cooperative Intelligent Transport (C-ITS) is subject to strong network effects, adoption of C-ITS is a key driver of its (societal) benefits. Therefore, this paper estimated the penetration rates of C-ITS equipped cars in the car park of Flanders, Belgium. Based on recent new Delegated Regulation, full penetration of C-ITS in the Flemish car park is expected later than twenty years after the Delegated Regulation of the European Commission and V2V mandate enter into force. In combination with Automated Driving, this can allow Flanders to reach zero traffic mortalities by 2050 , as envisioned by the European Commission.

Numbers on C-ITS adoption can be helpful for a number of stakeholders in Flanders. First, Flemish government and local governments can determine if the efforts of Europe need additional measures. Second, adoption rates act as a driver of (indirect) societal benefits, hence they serve as an input to cost-benefit analyses for governments planning to deploy Road Side Units (RSUs) in highway or urban setting. Finally, the Flemish government, but also technology providers and network operators, can use adoption numbers in the dimensioning of the technical solutions.

\section{REFERENCES}

[1] T. Rebbeck, J. Stewart, H.-A. Lacour, A. Killeen, A. Mason, D. Mcclure, and A. Dunoyer, "Socio-Economic Benefits of Cellular V2X," 2017.
[2] O. T. Move, "Towards a European road safety area ON THE MOVE for safer roads in Europe," 2010.

[3] E. Brivio and A. Perier, "Road Safety: Data show improvements in 2017 but renewed efforts are needed for further substantial progress," European Commission, Tech. Rep. April, 2018.

[4] B. Weyts and P. Muyters, "Bisconceptnota aan de regering - betreft: geconnecteerde en geautomatiseerde mobiliteit in Vlaanderen," no. VR 20180203 doc.0194/1bis, pp. 1-18, 2018.

[5] Vlaamse Regering, "Visie 2050 : Langetermijnstrategie voor Vlaanderen," Tech. Rep., 2016.

[6] A.-K. Itkonen and A. Perier, "European Commission - PRESS RELEASES - Press release - An EU strategy on cooperative, connected and automated mobility," 2016

[7] European Commission, "C(2019) 1789 final - Commission Delegated Reuglation of 13.3.2019 supplementing Directive 2010/40/EU of the European Parliament and of the Council with regard to the deployment and operational use of cooperative intelligent transport systems," 2019.

[8] N. Asselin-Miller, M. Biedka, G. Gibson, F. Kirsch, N. Hill, B. White, and K. Uddin, "Study on the Deployment of C-ITS in Europe: Final Report," Tech. Rep., 2016.

[9] European Commission, "A European strategy on Cooperative Intelligent Transport Systems, a milestone towards cooperative, connected and automated mobility,” European Commission, Tech. Rep., 2016.

[10] M. Makridis, K. Mattas, B. Ciuffo, M. A. Raposo, T. Toledo, and C. Thiel, "Connected and automated vehicles on a freeway scenario. Effect on traffic congestion and network capacity," in 7th Transport Research Arena TRA, 2018.

[11] IEEE - Standards Association, IEEE Standard for Information technology-Telecommunications and information exchange between systems Local and metropolitan area networks-Specific requirements Part 11: Wireless LAN Medium Access Control (MAC) and Physical Layer (PHY) Specifications. Institute of Electrical and Electronics Engineers, 2012, vol. 2012, no. March.

[12] ETSI, "Service requirements for V2X services (3GPP TS 22.185 version 14.3.0 Release 14)," Tech. Rep., 2017.

[13] M. Böhm, "C-Roads Position Paper: Radio frequencies designated for enhanced road safety in Europe - C-Roads position on the usage of the $5.9 \mathrm{GHz}$ band," 2017.

[14] D. L. Greene, "Why the Market for New Passenger Cars Generally Undervalues Fuel Economy," internationaltransportforum.org, 2010.

[15] R. van Maarseveen, J. Tijm, and P. Zwaneveld, "Stimuleer veiligste auto's: Baten verkeersveiligheid groot," Tech. Rep., 2018.

[16] Deloitte, "Great expectations models and consumer preferences," 2018.

[17] A. Arundel and R. Kemp, "Measuring eco-innovation," 2009.

[18] S. Zhou and M. A. Brown, "Smart meter deployment in Europe: A comparative case study on the impacts of national policy schemes," Journal of Cleaner Production, vol. 144, pp. 22-32, feb 2017.

[19] European Parliament, "Safer roads: new EU measures to reduce car accidents - News - European Parliament," 2019.

[20] European Commission, " $\operatorname{COM}(2018) 283$ final - On the road to automated mobility: An EU strategy for mobility of the future," European Commission, Brussels, Tech. Rep., 2018.

[21] E. Commission, "Cost-effectiveness analysis of Policy Options for the mandatory implementation of different sets of vehicle safety measures Review of the General Safety and Pedestrian Safety Regulations," Tech. Rep., 2018.

[22] K. E. Beyrouty, E. Löhr, T. Nokes, C. Brannigan, S. Levin, M. Biedka, H. Figg, and N. Asselin-Miller, "Support study for Impact Assessment of Cooperative Intelligent Transport Systems," Tech. Rep., 2018.

[23] S. Scheibe, "With the aim of increasing safety in road traffic, volkswagen will enable vehicles to communicate with each other as from 2019," 2017, [Online; accessed 7-March-2019].

[24] B. Lyons, "Toyota and lexus to launch technology to connect vehicles and infrastructure in the u.s. in 2021," 2018, [Online; accessed 7-March2019].

[25] S. Abuelsamid, "Ford breaks with auto rivals by committing to c-v2x vehicle communications tech," 2019, [Online; accessed 7-March-2019].

[26] European Commission, "C-ITS Platform Phase 2," 2017.

[27] L. Feng, "NGMN Alliance V2X White Paper," 2018.

[28] European Commission, "DIRECTIVE 2014/94/EU OF THE EUROPEAN PARLIAMENT AND OF THE COUNCIL - of 22 October 2014 - on the deployment of alternative fuels infrastructure -," 2014. 